\title{
Morfologia de espécies medicinais de boldo cultivadas no Brasil
}

\author{
Morphology of medicinal species of cultivated boldo in Brazil \\ Morfología de especies medicinales de boldo cultivadas en Brasil
}

Recebido: 06/05/2021 | Revisado: 16/05/2021 | Aceito: 18/05/2021 | Publicado: 04/06/2021

\author{
José Martins Fernandes \\ ORCID: https://orcid.org/0000-0002-8264-5085 \\ Universidade do Estado de Mato Grosso, Brasil \\ E-mail: jose.martins@unemat.br \\ Célia Regina Araújo Soares Lopes \\ ORCID: https://orcid.org/0000-0002-2123-0425 \\ Universidade do Estado de Mato Grosso, Brasil \\ E-mail: soaresia@unemat.br \\ Anderson Alex Sandro Domingos de Almeida \\ ORCID: https://orcid.org/0000-0003-3816-533X \\ Universidade do Estado de Mato Grosso, Brasil \\ E-mail: anderson.almeida@unemat.br
}

\begin{abstract}
Resumo
Para o Brasil, foram obtidos na literatura oito nomes de espécies conhecidas como boldo, quase todas com funções medicinais relacionadas ao estômago e fígado. $\mathrm{O}$ trabalho teve como objetivo apresentar diagnoses morfológicas para as espécies conhecidas como boldo, cultivadas no Brasil, bem como uma chave para identificação, figuras, comentários taxonômicos e medicinais. Foi baseado em cinco coletas férteis de boldo no município de Alta Floresta, Mato Grosso, e 47 exsicatas digitalizadas em bancos de dados online, provenientes de coletas realizadas no Brasil, totalizando 52 amostras examinadas. As diagnoses foram realizadas a partir de caracteres vegetativos e reprodutivos no Laboratório de Morfologia Vegetal, no Herbário da Amazônia Meridional (UNEMAT). São apresentadas três espécies de boldo cultivadas no Brasil, com usos medicinais relacionados ao estômago e fígado: Gymnanthemum amygdalinum (Delile) Sch.Bip. ex Walp., Plectranthus barbatus Andr. e Plectranthus neochilus Schltr., diferenciadas quanto a filotaxia, formato das lâminas e margens foliares, coloração de tricomas glandulares, tipos de inflorescências e adensamento entre as flores em antese. Espécimes determinados nos herbários como Plectranthus ornatus Codd não correspondem a espécie, mas a P. neochilus. Plectranthus amboinicus (Lour.) Spreng. não foi considerada como boldo devido não ter usos relacionados ao estômago e fígado, nem conhecida em literaturas tradicionais como boldo. Com o uso da morfologia vegetal, revisões taxonômicas, e-taxonomy e referências de plantas medicinais foi possível apresentar espécies popularmente chamadas de boldo no Brasil e com nomes científicos válidos, bem como, questionar espécies com ocorrência e nome popular duvidosos.
\end{abstract}

Palavras-chave: Plantas medicinais; Plectranthus neochilus; Taxonomia.

\begin{abstract}
For Brazil, eight species names known as boldo were obtained in the literature, almost all with medicinal functions related to stomach and liver. The work aimed to present morphological diagnoses for the species known as boldo, grown in Brazil, as well as a key for identification, figures, taxonomic and medicinal comments. The morphological study was based on five fertile boldo collects in the municipality of Alta Floresta, Mato Grosso, and 47 exsiccates digitized in online databases, from collects carried out in Brazil, totaling 52 samples examined. The diagnoses were made from vegetative and reproductive traits at the Plant Morphology Laboratory, at the Herbário da Amazônia Meridional (UNEMAT). Three species of boldo are grown in Brazil, with medicinal uses related to the stomach and liver: Gymnanthemum amygdalinum (Delile) Sch.Bip. ex Walp., Plectranthus barbatus Andr. and Plectranthus neochilus Schltr., differentiated in terms of phyllotaxis, blade shape and leaf margins, coloration of glandular trichomes, types of inflorescences and densification between flowers in anthesis. Specimens determined in herbariums such as Plectranthus ornatus Codd do not correspond to the species, but $P$. neochilus, due to the size of the corolla that does not exceed $2 \mathrm{~cm}$ in length. Plectranthus amboinicus (Lour.) Spreng. it was not considered as boldo because it has no uses related to the stomach and liver, nor known in traditional literature as boldo. With the use of plant morphology, taxonomic reviews, e-taxonomy and references of medicinal plants it was possible to present the species popularly called boldo in Brazil and with valid scientific names, as well as to question species with dubious occurrence and popular name.
\end{abstract}

Keywords: Medicinal plant; Plectranthus neochilus; Taxonomy. 


\begin{abstract}
Resumen
Para Brasil, encontramos en la literatura ocho especies conocidas como boldo, casi todas con funciones medicinales relacionadas con estómago e hígado. El objetivo de este estudio fue presentar diagnósticos morfológicos para las especies conocidas como boldo, cultivadas en Brasil, así como una clave para identificación, figuras, comentarios taxonómicos y medicinales. El estudio morfológico estubo basado en cinco colecciones de boldo fértiles en el municipio de Alta Floresta, Mato Grosso, y 47 exsicatas digitalizadas en bases de datos virtuales de colecciones realizadas en Brasil, totalizando 52 muestras examinadas. Los diagnósticos fueron realizados en el Laboratorio de Morfología de Plantas, del Herbario de la Amazônia Meridional (HERBAM) con base en caracteres vegetativos y reproductivos. Se presentan tres especies de boldo cultivadas en Brasil, y con usos medicinales relacionados con estómago e hígado: Gymnanthemum amygdalinum (Delile) Sch.Bip. ex Walp., Plectranthus barbatus Andr. y Plectranthus neochilus Schltr., diferenciado en términos de filotaxia, forma y margen de la hoja, coloración de tricomas glandulares, tipos de inflorescencias y densificación entre flores en antesis. Los especímenes determinados en herbarios como Plectranthus ornatus Codd no corresponden a la especie, sino a P. neochilus. Plectranthus amboinicus (Lour.) Spreng. no fue considerado boldo por no tener usos relacionados con estómago e hígado, siendo desconocido como boldo segun literatura tradicional. Con el uso de morfología vegetal, revisiones taxonómicas, etaxonomía y referencias de plantas medicinales fue posible presentar tres especies llamadas popularmente boldo en Brasil y con nombres científicos válidos, así como cuestionar especies con ocurrencia dudosa y nombre popular.
\end{abstract}

Palabras clave: Planta medicinal; Plectranthus neochilus; Taxonomía.

\title{
1. Introdução
}

A taxonomia de plantas medicinais pode ser considerada complexa, levando em consideração a quantidade de espécies vegetais conhecidas no mundo, a introdução constante de espécies exóticas influenciada por diferentes culturas e saberes, as mudanças taxonômicas em decorrência dos trabalhos filogenéticos moleculares, o número elevado de sinônimos disponíveis e, principalmente, poucos especialistas em identificação botânica.

A primeira etapa da validação de uma planta medicinal está no levantamento dos dados botânicos da espécie, que inclui o reconhecimento nos níveis de família, gênero e espécie (Vasconcelos, et al., 2005; Félix-Silva, et al., 2012; Leal-Costa, et al., 2018). Estas informações têm por finalidade garantir o uso seguro por parte do usuário de plantas medicinais (LealCosta, et al., 2018). O nome popular também pode contribuir no processo da identificação botânica, com cautela, retratando culturas e espaços geográficos, mas devendo levar em consideração a fonte da informação popular.

A Organização Mundial da Saúde reconhece que grande parte da população dos países em desenvolvimento, incluindo o Brasil, depende da medicina tradicional para sua atenção primária, tendo em vista que $85 \%$ utilizam plantas medicinais (Brasil, 2016) como fonte de recurso para solucionar problemas da saúde. No Brasil, alguns estados e municípios vêm realizando nas duas últimas décadas a implantação de Programas de Fitoterapia na atenção primária, com o intuito de suprir as carências medicamentosas de suas comunidades (Souza, et al., 2013).

No Brasil, são várias as abordagens nas pesquisas de plantas medicinais, como em etnobotânica (Amorozo \& Gély, 1988; Fernandes, et al., 2014; Cruz, et al., 2017; Pereira \& Coelho-Ferreira, 2017; Dluzniewski \& Müller, 2018; Mera, et al., 2018; Albuquerque, et al., 2019; Silva, et al., 2019; Pasa, et al., 2019), formas de cultivo, processamento (Rodrigues, 2004; Morais, et al., 2018), comercialização (Souza, et al., 2012; Brasil, 2016; Lima, et al., 2016), conservação (Martinelli \& Moraes, 2013) e como recurso didático para alunos do Ensino Básico (Andrade, et al., 2021; Castro, et al., 2021). Dentre as obras recentes que apresentam dados das espécies medicinais no Brasil, está o livro "Plantas Medicinais no Brasil - nativas e exóticas", com informações botânicas para 345 espécies (Lorenzi \& Matos, 2008), dentre elas, várias popularmente chamadas entre os brasileiros de "boldo", todas usadas para enfermidades do estômago e fígado.

É elevado o número de espécies medicinais popularmente chamadas de "boldo" no Brasil, são elas: Gymnanthemum amygdalinum (Delile) Sch.Bip. ex Walp., Peumus boldus Molina, Plectranthus amboinicus (Lour.) Spreng., Plectranthus barbatus Andr., Plectranthus grandis R. Willemse, Plectranthus neochilus Schltr., Plectranthus ornatus Codd e Vernonia condensata Baker (Lorenzi \& Matos, 2002; Lorenzi \& Matos, 2008; Mariano, 2015; Pereira \& Coelho-Ferreira, 2017), 
totalizando oito espécies, que remetem à algumas perguntas. Todas as espécies são válidas? Todas as espécies chamadas de "boldo" são usadas para problemas do estômago e fígado? E, todas são cultivadas no Brasil?

Levando em consideração a complexidade na identificação de plantas medicinais, contrapondo com a vasta literatura sobre o assunto no Brasil, o trabalho tem como objetivo apresentar diagnoses morfológicas para as espécies de boldo, cultivadas no país, chave para identificação, figuras, comentários taxonômicos e farmacológicos.

\section{Metodologia}

O estudo morfológico foi realizado entre janeiro e abril de 2020, baseado em espécimes de boldo coletados no município de Alta Floresta (Figura 1), Mato Grosso, e exsicatas digitalizadas em bancos de dados online provenientes de coletas realizadas no Brasil. As coletas foram realizadas em quintais urbanos e rurais, além de áreas comerciais em Alta Floresta, com a retirada de 5 ramos com folhas, flores e/ou frutos de cada indivíduo e armazenadas em saco plástico, com dados botânicos e geográficos anotados em caderno de campo, posteriormente, levadas ao Herbário da Amazônia Meridional (HERBAM), da Universidade do Estado de Mato Grosso, para herborização conforme metodologia usual (Fidalgo \& Bononi, 1989).

Foram utilizadas quarenta e sete exsicatas digitalizadas como material complementar, provenientes de coletas realizadas em 40 municípios do Brasil (Figura 1), obtidas no Herbário Virtual Reflora (2020), Jabot (2020) e SpeciesLink (2020), por meio de consulta utilizando-se nomes científicos disponíveis na literatura; as identificações e/ou confirmações dos nomes científicos foram realizadas por taxonomista, com suporte de revisões taxonômicas (Codd, 1975; Swelankomo, et al., 2016) e livros especializados em plantas medicinais (Lorenzi \& Matos, 2002; Lorenzi \& Matos, 2008); a grafia e validade dos nomes científicos e abreviações dos nomes dos autores foram conferidas em sites especializados (Flora do Brasil, 2020; Tropicos, 2020; World Flora Online, 2021); e, a classificação das famílias botânicas, conforme o Grupo de Filogenia das Angiospermas (APweb, 2021).

As diagnoses morfológicas foram realizadas com materiais frescos e complementadas com imagens de exsicatas selecionadas para o estudo, fazendo uso de um estereomicroscópio, régua, papel milimetrado, seringa com agulha e lâmina de aço, com terminologia baseada em Radford et al. (1974) e Barroso et al. (1999). A estrutura das diagnoses foi baseada em Codd (1975), Fernandes (2007), Fernandes (2011) e Swelankomo et al. (2016).

As abreviações utilizadas nas diagnoses e materiais selecionados são: $\mathrm{m}=$ metro, alt=altura, $\mathrm{cm}=$ centímetro, compr=comprimento, $\mathrm{mm}=$ milímetro, $\mathrm{fl}=\mathrm{flor}$ e $\mathrm{fr}=$ fruto; todos os materiais coletados em Alta Floresta tiveram exsicatas montadas e incorporadas ao acervo do HERBAM, com número de tombo disponível no material selecionado; e, foi elaborado um mapa contemplando os municípios com espécimes incluídos no trabalho, utilizando-se o software ArcGIS $^{\circledR}$ 10.2.2, licença teste, com uso dos recursos do ArcMap para criar o mapa tendo como bases cartográficas WGS-84 e o sistema de projeção UTM. 
Figura 1. Municípios do Brasil com espécimes de boldo incluídos no estudo: 1 - Alta Floresta (MT); 2-40 - demais municípios com exsicatas digitalizadas, com nomes e números correspondentes nos materiais selecionados de cada espécie.

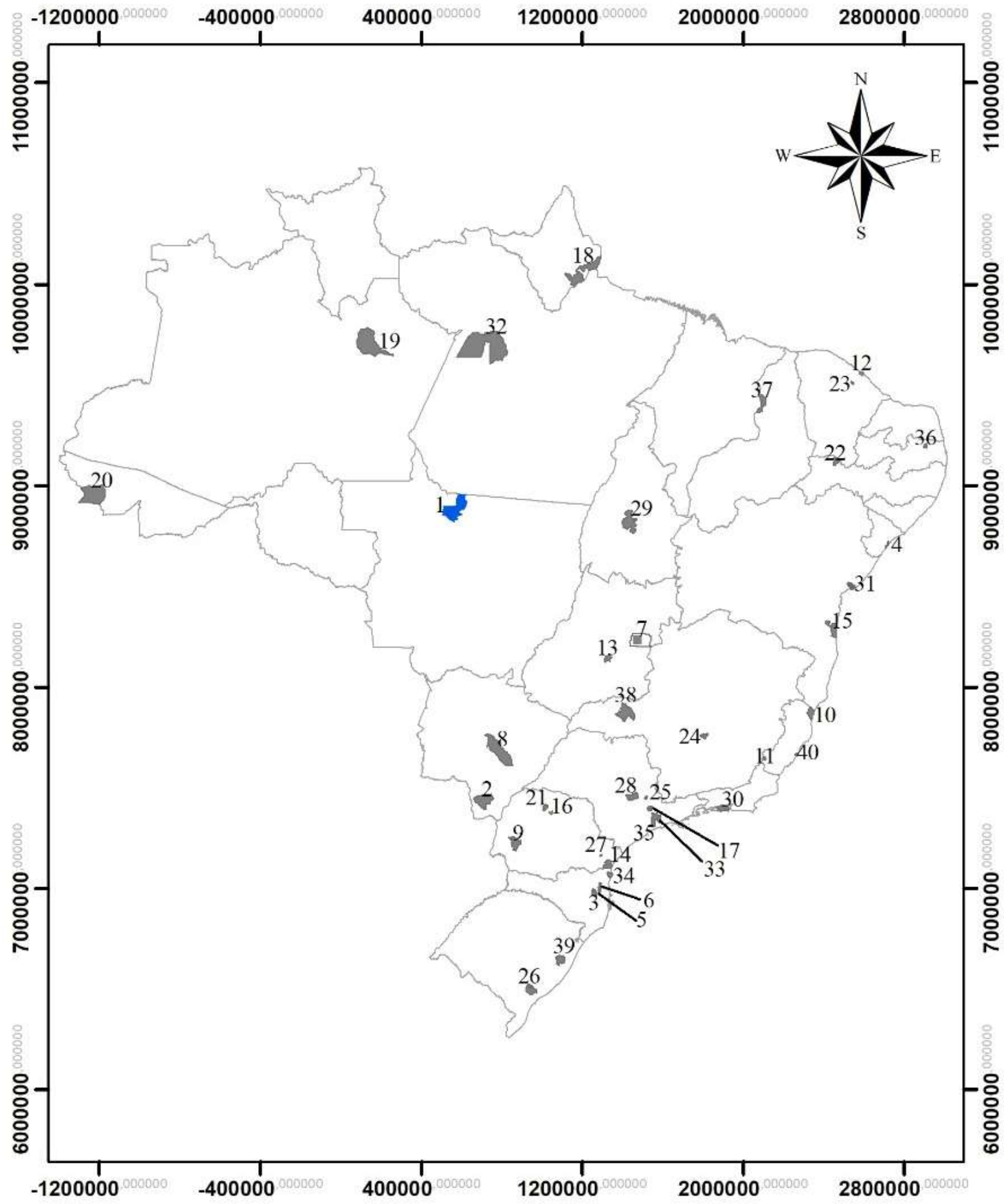

\section{Legenda}

\begin{tabular}{l}
\hline Alta Floresta-MT \\
\hline Outros Municípios \\
\hline$\quad$ Brasil
\end{tabular}

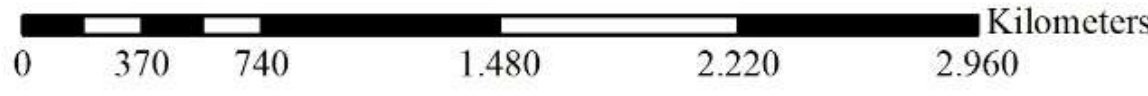

Sistema de Coordenadas UTM

Datum WGS84 Base:IBGE 2006

Elaborado por Almeida, A. A. S. D., 2020 


\section{Resultados}

O trabalho apresenta o estudo morfológico para três espécies de boldo cultivadas no Brasil, são elas Gymnanthemum amygdalinum (Asteraceae), Plectranthus barbatus e Plectranthus neochilus (Lamiaceae), e podem ser distinguidas por caracteres vegetativos e reprodutivos apresentados na chave a seguir.

\section{Chave para identificação de três espécies de boldo cultivadas no Brasil}

1. Folhas alternas; unidade de inflorescência em capítulo, reunidas em capitulescencias corimbiformes; flores com papilhos Gymnanthemum amygdalinum

1'. Folhas opostas; unidade de inflorescência em cimeira, reunidas em verticilastros; flores sem papilhos

2. Subarbustos eretos; folhas ovadas a elípticas, margem crenada a denteada em toda a margem, face abaxial com tricoma glandular verde-amarelado Plectranthus barbatus

2'. Ervas prostradas; folhas obovadas, margem crenada apenas na metade superior, face abaxial com tricoma glandular laranja

Plectranthus neochilus

Gymnanthemum amygdalinum (Delile) Sch.Bip. ex Walp., Repert. Bot. Syst. 2: 948. 1843 (Sinônimo: Vernonia condensata Baker, J. Bot. 8: 202. 1875).

Figura 2

Arbustos 2-4 m alt.; ramos jovens cilíndricos, densamente tomentosos, tricomas glandulares presentes, sésseis, cabeça secretora diminuta, incolor. Estípulas ausentes. Folhas alternas, simples, pecíolo 2,7-8 cm compr., subcilíndrico, esparso tomentoso, tricomas glandulares presentes, lâmina 12-25 × 5,5-11 cm, elíptica, base atenuada, ápice agudo a obtuso, faces adaxial e abaxial glabrescentes, tricomas glandulares presentes, incolores, margens sinuosas a levemente serradas. Unidades de inflorescência em capítulos, reunidas em capitulescencias corimbiformes, pedunculada, pedúnculo $2-6 \mathrm{~mm}$ compr., 3-5 brácteas, 1,3-1,4 mm compr., 5 ciclos de brácteas involucrais, 3-4,2 × 1,3-1,8 mm, elípticas, glabras, margem ciliada. Flores homomórficas, 14-18 flores por capítulo, actinomorfas, não vistosas, pentâmeras; papilhos 5,5-6 mm compr., esbranquiçados, tricomas presentes; corola 7-7,6 $\mathrm{mm}$ compr., tubular, branca, tubo 5-5,3 $\mathrm{mm}$ compr., tricomas glandulares presentes, incolores, lobos 2-2,3 mm compr., glabros; 5 estames, epipétalos, sinanteros, filetes 1,8-2 mm compr., anteras 2,8-3 mm compr., rimosas; ovário ínfero, 1,8-3 mm compr., estrigoso, tricomas glandulares presentes, estilete 5-5,5 mm compr., glabro, estigma 2,2-2,5 mm compr., bífido. Fruto cipsela, 3-3,5 mm compr., 10-nervado, papilhos persistentes, cor palha.

Material selecionado. BRASIL. Acre: Marechal Thaumaturgo (Figura 1, $\mathrm{n}^{\circ}$ 20), Reserva Extrativista do Alto Juruá, 19/07/1992, fr., L.C. Ming 319 (foto NY!). Amapá: Macapá (Figura 1, nº 18), Curió de Dentro, 05/12/2001, fr., A. Stipanovich 51 (foto EAC!). Amazonas: Manaus (Figura 1, n 19), SUFRAMA, s.d., fl., M.H. Nee 43008 (foto NY!). Bahia: Ilhéus (Figura 1, no 15), Mata da Esperança, 21/09/2000, fr., L.C. B. Costa 155 (foto CEPEC!). Ceará: Fortaleza (Figura 1, no 12), Parque São João, 10/11/1997, fr., A.S.F. Castro (foto EAC 26046!). Distrito Federal: Brasília (Figura 1, nº 7), Bacia do Riacho, 27/09/2000, fl., A.E. Ramos 1451 (foto HEPH!). Espírito Santo: Conceição da Barra (Figura 1, nº 10), 09/07/2011, fl., M.G.S. Fink 53 (foto RB!). Goiás: Goiânia (Figura 1, nº 13), 25/08/2014, H.E. Amaral e J.C. Freire 34 (foto UFG!). Mato Grosso: Alta Floresta (Figura 1, $\mathrm{n}^{\circ}$ 1), entrada do Buriti Empreendimentos, ao lado do Vilsinho Tratores, 04/04/2020, fl., J.M. Fernandes 1597 (HERBAM 26581), 22/04/2020, fl., fr., J.M. Fernandes 1605 (HERBAM 26582). Mato Grosso do Sul: Campo Grande (Figura 1, n 8), 08/09/2004, fr., W.S. Garcéz 146 (foto HUFU!). Minas Gerais: Pará de Minas (Figura 1, nº 24), 08/02/1976, fr., S.B. Jones 74 (foto RB!). Pará: Santarém (Figura 1, no 32), quintal PA-370, 27/07/2018, f1., A.C. Costa 01 (foto HSTM!). Paraná: Maringá (Figura 1, nº 21), Campus da UEM, 05/12/1984, fl., fr., Marli (HUEM 1923!). Pernambuco: 
Moreilândia (Figura 1, $\mathrm{n}^{\circ}$ 22), Serra do Cotolé, 06/07/2014, U.M. Mascena 36 (foto EAC!). São Paulo: Piracicaba (Figura 1, $\mathrm{n}^{\circ}$ 28), s.d. fl., P. Milan 06 (foto ESA!). Santa Catarina: São Francisco do Sul (Figura 1, no 34), 24/06/2012, fl., M.S. Marques (foto FLOR 50702!). Sergipe: Aracaju (Figura 1, nº 4), Bairro Santo Antônio, 08/09/1987, fl., fr., C.M. Moura (foto VIC 10189!). Tocantins: Porto Nacional (Figura 1, n 29), Alta da Colina, 30/11/1999, fr., E.M. Silva (foto BOTU 24160!).

Figura 2. Gymnanthemum amygdalinum - A, hábito; B, folha; C, capítulos; D, flor.
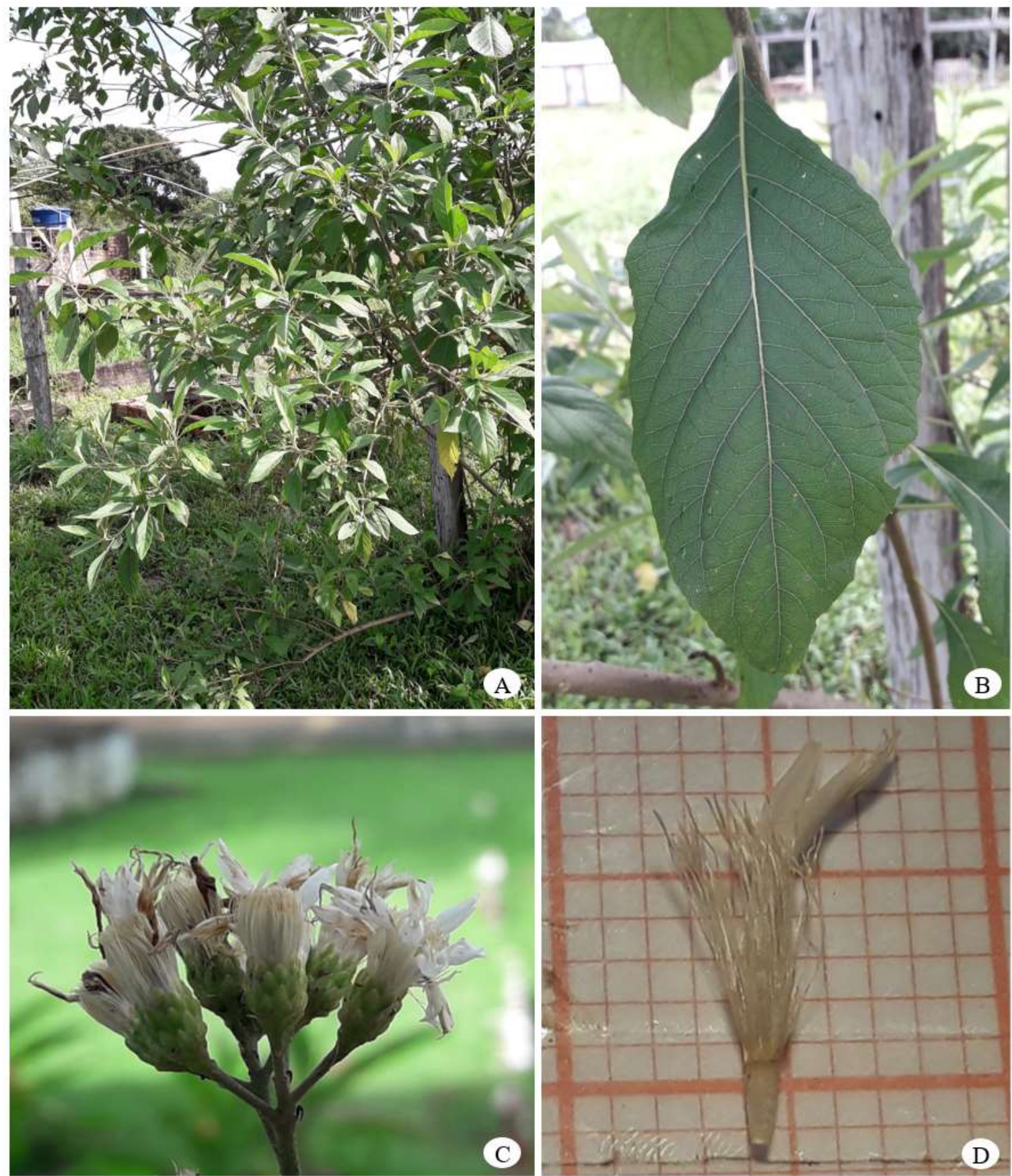

Fonte: J. M. Fernandes (A-D).

Plectranthus barbatus Andrews, Bot. Repos. 9: t. 594.1809 (Sinônimos: Coleus barbatus (Andrews) Benth. ex G. Don, Hort. Brit. 15483, fig. 4C. 1830, Plectranthus grandis R. Willemse, Blumea 25: 509. 1979).

Figura 3 
Subarbustos 0,5-1,5 m alt., eretos; ramos jovens subcilíndricos, tricomas curto-vilosos e longo-seríceos, tricomas glandulares sésseis, cabeça secretora verde-amarelada. Estípulas ausentes. Folhas opostas, simples, pecíolo 1,4-3 cm compr., canaliculado, lâmina 7,5-13,5 × 4-7 cm, ovada a elíptica, base atenuada, ápice obtuso, face adaxial com tricomas longos, seríceos, face abaxial vilosa, tricomas glandulares densos, cabeça secretora verde-amarelada, margem crenada a denteada. Unidades de inflorescência em cimeiras, laxas com flores em antese, reunidas em verticilastros, $18-25 \mathrm{~cm}$ compr., brácteas $1,2-1,5 \times 0,7-1 \mathrm{~cm}$, ovadas, serícea, Flores pediceladas, pedicelo $3-5 \mathrm{~mm}$ compr., tricomas glandulares estipitados presentes, cabeça secretora diminuta, translúcida, zigomorfas, vistosas, pentâmeras; cálice 4,8-7 mm compr., gamossépalo, tricomas velutinos no ápice, tubo 1,8-2 mm compr., lobo superior 4-5 mm compr., ovado, lobos laterais e inferiores 3-3,5 mm compr., lanceolados a estreitamente triangulares; corola 2,4-2,7 cm compr., bilabiada, gamopétala, azul, tricomas glandulares presentes, tubo 1-1,1 cm compr., fortemente geniculado, 4 lobos superiores, 9-13 mm compr., 1 lobo inferior, 20-23 mm compr., cimbiforme; androceu gamostêmone, didínamo, filetes 4-7 mm compr., curvos, inclusos, anteras $1 \mathrm{~mm}$ compr., rimosas; ovário súpero, 1-1,5 mm compr., glabro, estilete 3-3,4 cm compr., estigma curtamente bífido. Fruto esquizocarpo, 1,4-1,8 × 1,7-2 mm, transversalmente oblongo, mericarpos 0,9-1 ×0,5-0,6 mm, elipsoides.

Material selecionado. BRASIL. Bahia: Salvador (Figura 1, no 31), Praia Grande, Comunidade Quilombola, 20/02/2013, fl., E.M.G.B. Silva 120 (foto HUEFS!). Ceará: Fortaleza (Figura 1, nº 12), $20 \mathrm{Km}$ de Crato, 12/11/1999, fr., R.L. Albuquerque (foto EAC 28599!). Distrito Federal: Brasília (Figura 1, no 7), Núcleo Bandeirante, 09/10/1991, fr., R.F. Vieira 913 (foto CEN!). Espírito Santo: Vitória (Figura 1, nº 40), Parque Municipal de Tabuazeiro, 24/04/2014, fr., A.S. Borges (foto VIES 35080!). Mato Grosso: Alta Floresta (Figura 1, $\mathrm{n}^{\circ}$ 1), Bairro Jardim Universitário, em frente a Oficina Tropical, 04/04/2020, fl., J.M. Fernandes 1596 (HERBAM 26580), Bairro Jardim Universitário, em frente ao ponto dos boiadeiros, 04/04/2020, fl., fr., J.M. Fernandes 1595 (HERBAM 26579), 22/04/2020, fr., J.M. Fernandes 1606 (HERBAM 26583). Mato Grosso do Sul: Amambai (Figura 1, no 2), centro, 03/10/1993, fl., P.C. Palhano 28 (foto SPF!). Minas Gerais: Uberlândia (Figura 1, nº 38), Sobradinho, 04/08/2004, fl., J.M. Barbosa 105 (foto HUFU!). Paraíba: Solânea (Figura 1, no 36), região do Curimataú, 09/11/2000, fl., T. Grisi 101 (foto HUEFS!). Paraná: Guaratuba (Figura 1, nº 14), São João, 10/11/1974, fl., G. Hatschbach 34847 (foto MBM!). Piauí: Teresina (Figura 1, n 37), Vila São Francisco, 30/03/2003, fl., A. Carvalho 281 (foto HUEFS!).

Rio Grande do Sul: Viamão (Figura 1, no 39), terra Indígena Guarani Cantagalo, 17/02/2018, fl., J.H. Andrade 18 (foto FLOR!). Santa Catarina: Apiúna (Figura 1, $\mathrm{n}^{\circ}$ 3), Neisse Central, 22/10/2012, fl., B. Tribess 30 (foto FURB!). São Paulo: São Paulo (Figura 1, n 35), 05/09/1989, fl., B. Djuragin (foto UNIP 3701!). Sergipe: Aracajú (Figura 1, no 4), Unidade de Saúde Manoel de Souza Pereira, 25/09/2015, fl., R.S. Andrade 60 (foto ASE!). 
Research, Society and Development, v. 10, n. 6, e42910615824, 2021

(CC BY 4.0) | ISSN 2525-3409 | DOI: http://dx.doi.org/10.33448/rsd-v10i6.15824

Figura 3. Morfologia de Plectranthus barbatus: A, hábito; B, folha; C, tricomas glandulares na face abaxial; D, inflorescência com ramos jovens; E, botões e brácteas; F, flor.

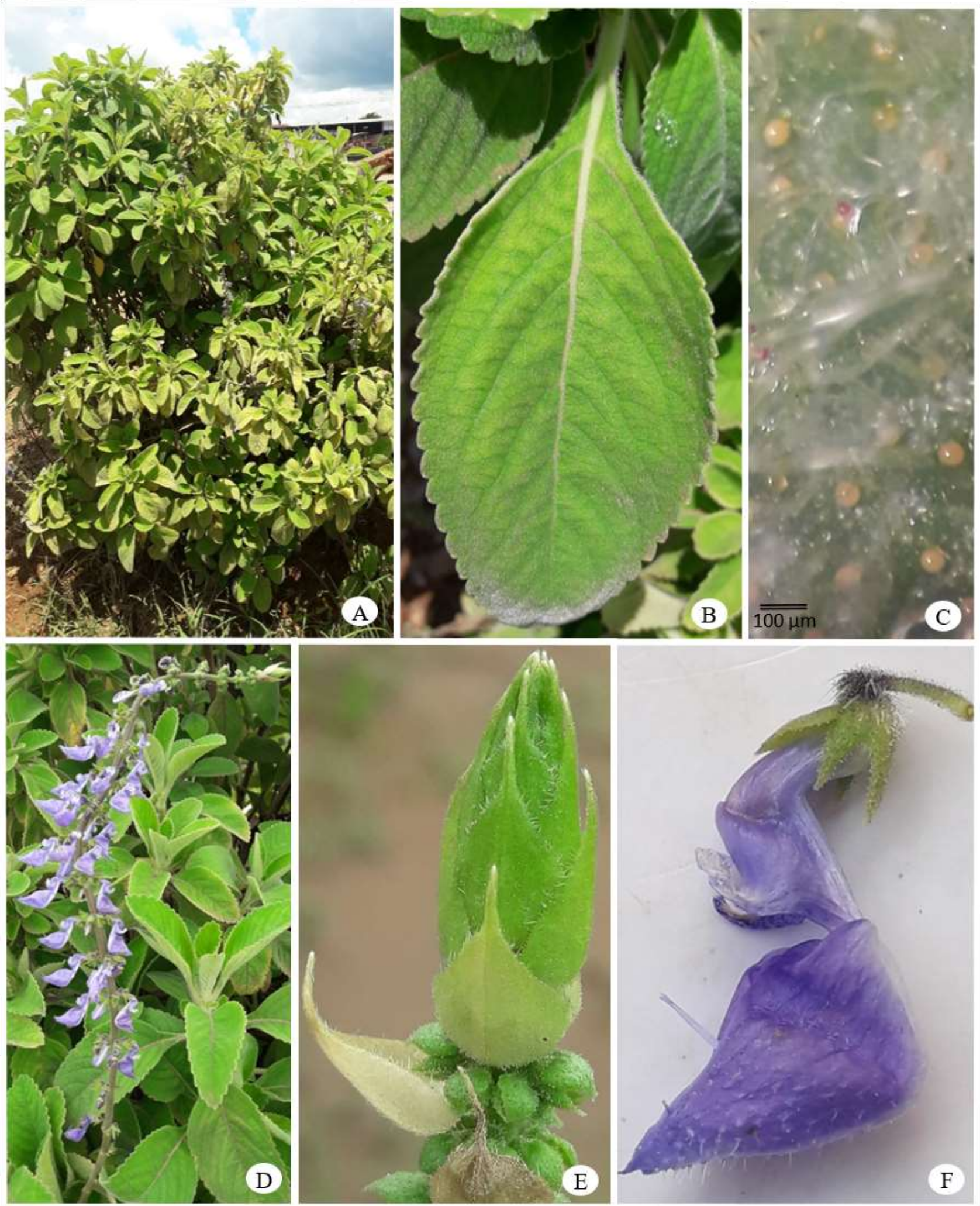

Fonte: J. M. Fernandes (A-F).

Plectranthus neochilus Schltr., J. Bot. J. Bot. 34: 394. 1896.

Figuras 4-5 
Ervas 20-50 cm alt., prostradas; ramos jovens cilíndricos, densamente seríceos, tricomas glandulares presentes, cabeça secretora laranja. Estípulas ausentes. Folha oposta, simples, pecíolo 4-6 mm compr., aplainado, seríceo, tricomas glandulares presentes, lâmina 4,3-5 × 2,4-2,6 cm, obovada, base atenuada, ápice obtuso a arredondado, faces adaxial e abaxial seríceas, tricomas glandulares presentes, laranja, margem da metade inferior inteira e margem da metade superior crenada, 4-6 pares de lobos. Unidades de inflorescência em cimeiras, congestas com flores em antese, reunidas em verticilastros, 4,5-22 cm compr., brácteas 1-1,2 $\times$ 0,7-0,9 mm, ovadas. Flores pediceladas, pedicelo 1-1,5 mm compr., indumento não observado, zigomorfas, vistosas, pentâmeras; cálice 3-3,3 mm compr., gamossépalo, indumento não observado, tubo 1,4-1,8 mm compr., lobos 1,8-2 mm compr.; corola 1,4-1,8(-2) cm compr., gamopétala, bilabiada, branca-azulada, indumento não observado, tubo 0,4-0,7 cm compr., 4 lobos superiores, 0,5-0,65 mm compr., 1 lobo inferior, 1-1,2 cm compr., cimbiforme; androceu gamostêmone, didínamo, filetes 10-14 mm compr., curvos, anteras rimosas; gineceu e fruto não observados.

Material examinado. BRASIL. Ceará: Pacoti (Figura 1, no 23), pernambuquinho, 31/07/2002, fl., fr., J.A. Portela (foto EAC 31929!). Distrito Federal: Brasília (Figura 1, n 7), Horto do Hospital das Forças Armadas, 20/11/1999, fl., fr., N.L.N. Júnior (foto EAC 28659!). Espirito Santo: Divino de São Lourenço (Figura 1, $\mathrm{n}^{\circ}$ 11), Caparaó, 17/05/2007, fl., T. Chimalli (foto VIES 27545!). Paraná: Cascavel (Figura 1, n 9), Campus da Universidade Estadual Oeste do Paraná, 28/08/2018, fl., fr., R.M. Racki 01 (foto UNOP!); Jandaia do Sul (Figura 1, no 16), 10/10/2005, fl., fr., P.H.L. Ramos (foto MBM 14700!); Maringá (Figura 1, $\mathrm{n}^{\mathrm{o}}$ 21), Horto de Plantas Medicinais Irenice Silva, Campus da Universidade de Maringá, 17/08/2005, fl., A.M. Janunzzi 124 (foto HUEM!); Pinhais (Figura 1, $\mathrm{n}^{\circ}$ 27), mundo do campo, PNFM, 27/05/2008, fl., V.L.A.R Freitas 18 (foto MBM!). Rio de Janeiro: Rio de Janeiro (Figura 1, n 30), Jardim Botânico do Rio de Janeiro, canteiro 21, 13/07/2015, fl., J.R. Mattos 134 (foto RB!). Rio Grande do Sul: Pelotas (Figura 1, n 26), Embrapa Clima Temperado, Horto Medicinal, 12/2002, fl., M. Couto (foto ECT 1408!). Santa Catarina: Blumenau (Figura 1, no ${ }^{\circ}$ ), Nova Rússia, 12/01/2004, fl., fr., F. Bosio (foto FURB 4104!); Apiúna (Figura 1, no 3), Vargem Grande, 26/10/2002, fl., B. Tribess 01 (foto FURB!); Ascurra (Figura 1, $\mathrm{n}^{\circ}$ 5), 01/10/2007, fl., L. Meyer (foto FURB 1982!); São Francisco do Sul (Figura 1, no 34), Ilha Guaraqueçaba, 16/09/2004, fl., $P$. Campregher 09 (foto FURB!). São Paulo: Atibaia, 33 km de Jundiaí, 29/11/2016, fl., fr., I.M. Galbiatti \& A.C.H.F Sawaya 04 (foto UEC 193816!); Jundiaí (Figura 1, n 17), 18/09/2007, fl., J.A. Lombardi 6905 (foto ESA!); Paulínia (Figura 1, n 25), 01/09/2007, fl., fr., G.A. Ogasawara 09 (foto ESA!); Santo André (Figura 1, nº 33), Vila de Paranapiacaba, 14/01/2014, fl., fr., B.M. Mourad 16 (foto HUFABC!); São Paulo (Figura 1, nº 35), Moema, Parque Ibirapuera, portão 07, 08/09/2001, fl., C.M. Izumisawa (foto PMSP 6593!).

A diagnose de Plectranthus neochilus foi realizada a partir de 18 espécimes examinados, no entanto, cinco deles estão determinados erroneamente como Plectranthus ornatus, coletados por G.A. Ogasawara 09 (foto ESA!), J.A. Portela (foto EAC 31929!), C.M. Izumisawa (foto PMSP 6593!), B.M. Mourad 16 (foto HUFABC!) e N.L.N. Júnior (foto EAC 28659!), incluídos no presente trabalho como P. neochilus. Uma comparação entre P. neochilus e Plectranthus ornatus está disponível na Tabela 1. 
Research, Society and Development, v. 10, n. 6, e42910615824, 2021

(CC BY 4.0) | ISSN 2525-3409 | DOI: http://dx.doi.org/10.33448/rsd-v10i6.15824

Figura 4. Plectranthus neochilus: A, hábito; B, ramo jovem com folhas; $\mathrm{C}$, tricomas glandulares na face abaxial da lâmina foliar; D, ramos com inflorescências; E, parte de uma inflorescência com brácteas e flores.
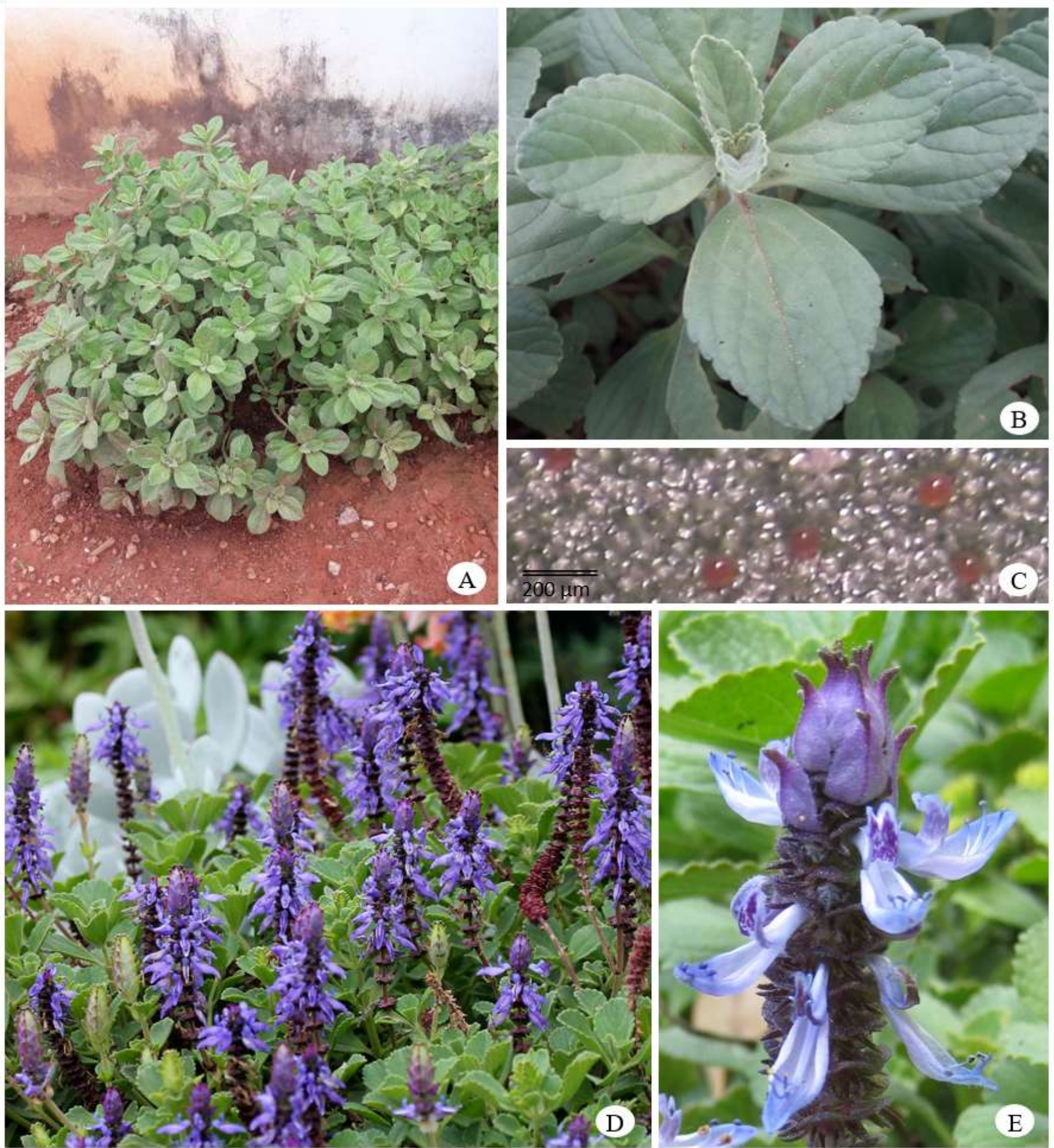

Fonte: J. M. Fernandes (A-C), Annie’s Annuals and Perennials (D) e Wikipedia ${ }^{\circledR}$ (E). 
Research, Society and Development, v. 10, n. 6, e42910615824, 2021

(CC BY 4.0) | ISSN 2525-3409 | DOI: http://dx.doi.org/10.33448/rsd-v10i6.15824

Figura 5. Imagens de exsicatas de Plectranthus neochilus: A, folhas e inflorescências com flores e botões; B, ramos com folhas e inflorescências com flores e botões.

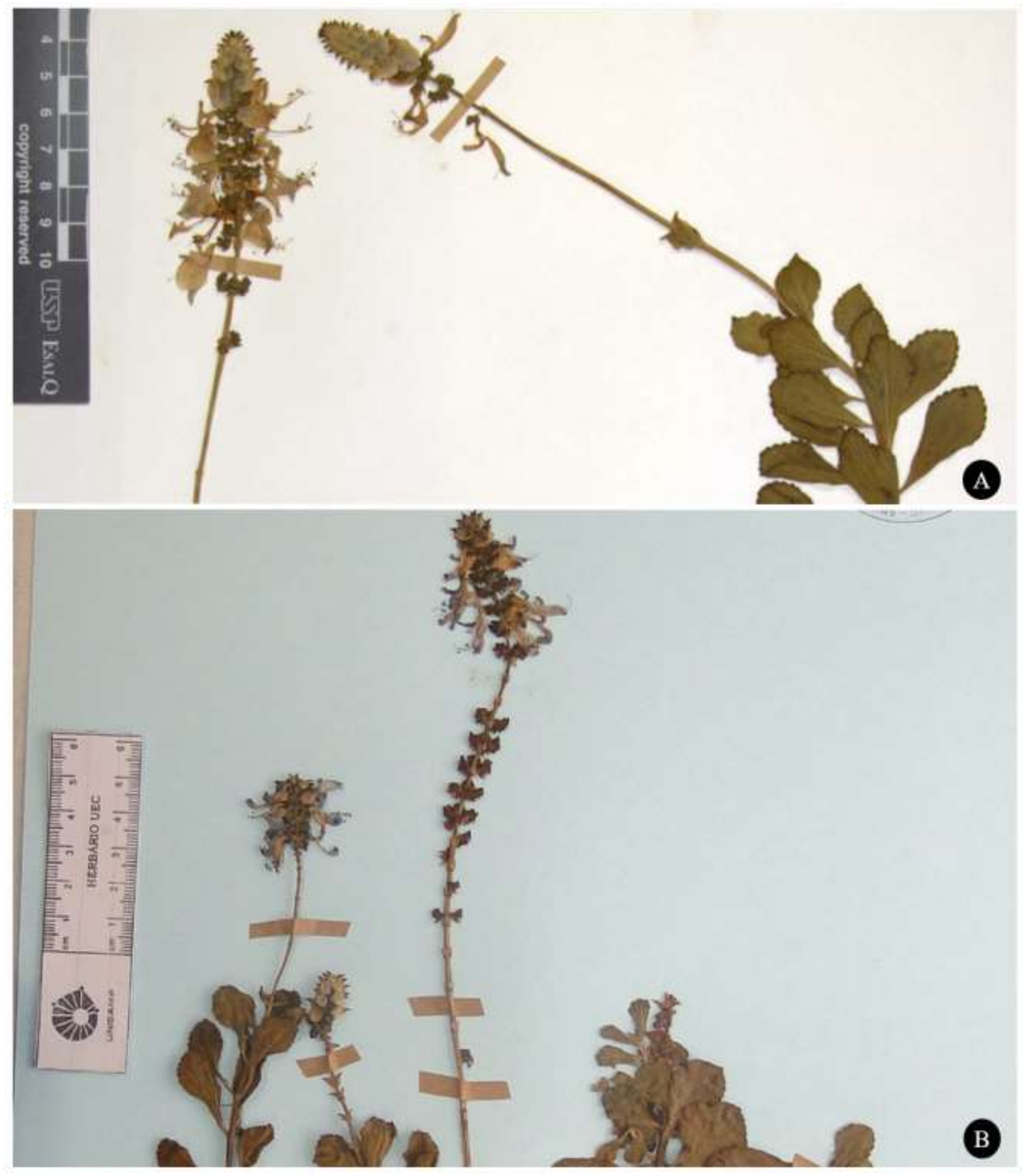

Fonte: ESA - Herbário da Escola Superior de Agricultura Luiz de Queiroz, coleta de J.A. Lombardi 6905 (A); UEC - Herbário da Universidade Estadual de Campinas, coleta de I.M. Galbiatti \& A.C.H.F Sawaya 04 (B). 
Tabela 1. Comparação morfológica entre Plectranthus neochilus e Plectranthus ornatus.

\begin{tabular}{l|l|l}
\hline Caracteres & \multicolumn{1}{|c}{ P. neochilus ${ }^{1}$} & \multicolumn{1}{c}{${\text { P. } \text { ornatus }^{2}}^{-1}$} \\
\hline Lâmina foliar & $\begin{array}{l}\text { obovada, metade superior crenada, 4-6 } \\
\text { pares de lobos }\end{array}$ & obovada, metade superior crenada, 4-6 pares de lobos \\
\hline Tricoma glandular & laranja & laranja \\
\hline Inflorescência & pedúnculo: $2,5-8 \mathrm{~cm}$ compr. & - \\
\hline Cálice & Raque: $4,5-22 \mathrm{~cm}$ compr. & raque: $3-5(-9) \mathrm{cm}$ compr. \\
\hline Corola & $3-3,3 \mathrm{~mm}$ compr. & $3 \mathrm{~mm} \mathrm{compr.}$ \\
\hline
\end{tabular}

Fonte: ${ }^{1}$ Autores; ${ }^{2}$ Codd (1975).

\section{Discussão}

As três espécies medicinais de boldo, cultivadas no Brasil, são usadas para tratamento de distúrbios no estômago e fígado (Lorenzi \& Matos, 2008; Cruz, et al., 2017; Pereira \& Coelho-Ferreira, 2017; Dluzniewski \& Müller, 2018; Silva, et al., 2019), facilmente diferenciadas pelo hábito, filotaxia, coloração do tricoma glandular, tipo de inflorescência e presença ou ausência de papilho na flor. O agrupamento de espécies a partir do nome popular, como realizado no presente trabalho, foi apresentado por Ferreira et al. (2004), que reuniram as espécies madeireiras de Leguminosae conhecidas como angelim no Estado do Pará (Andira surinamensis, Dinizia excelsa, Hymenolobium excelsum, H. modestum, H. pulcherrimum, H. petraeum e Vatairea paraensis), oferecendo a caracterização morfológica das espécies; e, por Moutinho (2008), que agrupou as espécies de Lecythidaceae com potencialidade madeireira na Amazônia brasileira, chamadas de matá-matá (Eschweilera amazonica, E. coriacea, E. grandiflora, E. ovata e Lecythis idatimon), oferecendo caracterização anatômica, física e química das madeiras.

As espécies de boldo apresentaram em comum a presença de tricomas glandulares na face abaxial, mas distinguindose pela coloração verde-amarelado (P. barbatus), laranja ( $P$. neochilus) e incolor (G. amygdalinum). Segundo Alquini et al. (2004), os tricomas glandulares estão envolvidos com secreção de várias substâncias, como óleo, néctar, sal, resina, mucilagem, suco digestivo e água, com uma cabeça uni ou multicelular que pode apresentar grande variedade de forma e tamanho para o acúmulo da secreção, que pode variar conforme o grupo taxonômico. De acordo com Martins e Pastori (2004), muitas espécies aromáticas são usadas como medicinal devido a quantidade de óleo essencial presente nos tricomas glandulares.

A espécie Gymnanthemum amygdalinum é nativa em toda a África Tropical, estendendo-se a Botsuana e África do Sul, em pântanos e margens de rios entre 130 e 1065 metros de altitude (Swelankomo, et al., 2016). Foi introduzida no Brasil no período colonial pelos escravos trazidos da África, tornando-se amplamente cultivada em hortas e jardins domésticos no país, conhecida como boldo, alumã, aloma, aluman, alcachofra, figatil, boldo-de-goiás, heparém, boldo-chinês, boldo-goiano, boldo-japonês, boldo-baiano, árvore-do-pinguço e cidreira-da-mata (Lorenzi \& Matos, 2008).

Diferencia das outras duas espécies de boldo por apresentar folha alterna, inflorescência em capítulo e fruto do tipo cipsela com papilho persistente. Os capítulos dessa espécie possuem entre 16-17 flores, corola tubulosa, lobos com ápice obtuso, anteras com apêndice agudo, base sagitada, estilete glabro na base e cipselas oblongas, 10-costadas (Nakajima, 2020), semelhante ao encontrado no presente estudo. Quanto ao uso medicinal, a espécie é empregada na medicina popular para tratamento da dor de estômago, diarreia, gases intestinais, insuficiência hepática, inflamação da vesícula e cólica renal (Gois, et al., 2016; Pereira \& Coelho-Ferreira, 2017).

A espécie Plectranthus barbatus é nativa em áreas florestais e rochosas na Etiópia, Sudão, Tanzânia e Índia (Codd, 1975). Foi introduzida no Brasil, provavelmente trazida pelos portugueses da Índia, ainda no período colonial, por isso o nome 
boldo-do-reino, pois foi introduzida pelo Reino de Portugal (Lorenzi \& Matos, 2002; Bandeira, 2007). É conhecida como boldo, falso-boldo, boldo-brasileiro, boldo-do-reino, alum, boldo-nacional, malva-santa, malva-amarga, sete-dores, boldo-dejardim, boldo-do-brasil e folha-de-oxalá (Lorenzi \& Matos, 2008).

Pode ser diferenciada facilmente das outras espécies de boldo, no Brasil, por apresentar folhas ovadas a elípticas, com margens crenadas a denteadas, presença de tricomas glandulares verde-amarelados na face abaxial e inflorescências laxas com flores em antese. Os espécimes analisados no Brasil estão de acordo com as informações morfológicas apresentadas para a espécie na África (Codd, 1975).

A espécie é usada no tratamento de gastrite, na dispepsia, azia, mal-estar gástrico (estômago embrulhado), ressaca, estimulante da digestão e do apetite, fazendo o uso de folhas frescas maceradas ou o chá na forma de infusão (Lorenzi \& Matos, 2008). É uma das espécies medicinais mais citadas em estudos etnobotânicos no Brasil (Fernandes, 2002; Cruz, et al., 2017; Dluzniewski \& Müller, 2018; Silva, et al., 2019), cultivada em quintais urbanos e rurais, além de estabelecimentos comerciais em áreas urbanas.

Plectranthus neochilus é nativa na África do Sul, Botswana, Zâmbia e Zimbábue, em áreas de sub-bosque em floresta aberta e seca, vegetação rochosa e em pastagem (Schlechter, 1896; Codd, 1975). No Brasil, é chamada popularmente como boldo e boldo-gambá (Lorenzi \& Matos, 2002). Os espécimes observados para as regiões Norte e Centro-Oeste do Brasil, incluindo amostras do município de Alta Floresta, não apresentaram órgãos reprodutivas, provavelmente por questões climáticas, apenas para as regiões Nordeste, Sudeste, Sul e Distrito Federal do país.

A espécie é facilmente reconhecida por apresentar folhas obovadas, com margem inteira até a metade inferior e metade superior crenada, tricomas glandulares na face abaxial de coloração laranja e inflorescência congesta com flores em antese. Ficou evidente a variação no tamanho das inflorescências de $P$. neochilus, variando de tamanho no mesmo material, mas o tamanho da corola se manteve estável, raramente chegando a 2 centímetros de comprimento. Para materiais africanos as inflorescências estão entre 7-15 centímetros de comprimento e corola de 1,2 a 2 centímetros de comprimento (Codd, 1975).

A espécie é muito aromática e pertence ao grupo dos "boldos" com usos medicinais semelhantes a P. barbatus (Lorenzi \& Matos, 2002). Outros autores também enfatizam que suas folhas são utilizadas para o tratamento de insuficiência hepática e dores no estômago (Duarte \& Lopes, 2007).

Três espécies popularmente chamadas de boldo no Brasil não foram incluídas no presente trabalho. Plectranthus ornatus, também chamada de boldo e boldinho (Lorenzi \& Matos, 2008), não é confirmada para o Brasil, levando em consideração caracteres morfológicos. Os materiais examinados com a identificação de $P$. ornatus, nos dados online de herbário, correspondem a P. neochilus. Segundo Galbiatti (2019), vários pesquisadores que trabalham com plantas medicinais e, até mesmo com taxonomia no Brasil, se deparam com dúvidas na distinção entre P. ornatus e P. neochilus.

Plectranthus ornatus assemelha-se com $P$. neochilus por apresentar folhas obovadas com margens crenadas na metade superior e tricomas glandulares laranjas na face abaxial, mas diferencia-se por apresentar inflorescências entre 3-5(-9) centímetros de comprimento e corola entre 2-2,5 centímetros de comprimento, enquanto $P$. neochilus possui inflorescência entre 7-15 centímetros de comprimento e corola com 1,2-2 centímetros de comprimento (Codd, 1975). Nos espécimes examinados, apenas o tamanho da corola se manteve estável (1,4-1,8 cm compr.), raramente chegando a 2 centímetros de comprimento, que corresponde a $P$. neochilus, enquanto que o tamanho da inflorescência não separa os dois táxons, demonstrando fragilidade na circunscrição das duas espécies.

Plectranthus amboinicus não foi incluído no grupo dos boldos por não apresentar função relacionada ao estômago ou fígado, nem é chamada de boldo em referencias tradicionais, mas como hortelã-grande (Lorenzi \& Matos, 2008), contrariando uma única referência (Mariano, 2015). A espécie Peumus boldus (Monimiaceae), nativa no Peru, é comercializada no Brasil 
como boldo-verdadeiro para enfermidades do estômago e fígado, mas não é cultivada por limitações climáticas (Lorenzi \& Matos, 2008).

\section{Conclusão}

O trabalho apresenta o agrupamento das espécies medicinais de boldo utilizadas no tratamento de problemas do estômago e fígado, com as principais características morfológicas para distinção no Brasil. Também demonstra a possibilidade em rejeitar nome popular duvidoso, sem nenhuma relação no tratamento de enfermidades tradicionalmente conhecidas para o grupo dos "boldos", com o objetivo de dificultar o uso da planta de forma equivocada.

Dentre os materiais examinados foi possível constatar que a planta conhecida como boldo-gambá corresponde a Plectranthus neochilus, e não a Plectranthus ornatus.

Diante do exposto, fica evidente a importância das literaturas taxonômicas nas investigações de plantas medicinais, oferecendo suporte teórico para a identificação das espécies. Atualmente, literaturas clássicas como floras, monografias, livros e artigos estão digitalizadas e com fácil acesso na internet, por outro lado, as recentes também são de fácil acesso, como sites especializados que apresentam dados sobre riqueza, fitogeografia, imagem de exsicata e nomenclatura de planta.

\section{Referências}

Albuquerque, U. P., Nascimento, A. L. B., Soldati, G. T., Feitosa, I. S., Campos, J. L. A., Hurrell, J. A., Hanazaki, N., Medeiros, P. M., Silva, R. R. V., Ludwinsky, R. H., Júnior, W. S. F., \& Reyes-García, V. (2019). Ten important questions/issues for ethnobotanical research. Acta Botanica Brasilica, 33(2), 376-385. doi.org/10.1590/0102-33062018abb0331

Alquini, Y., Bona, C., Boeger, M. R. T., Costa, C. G., \& Barros, C. F. (2004). Epiderme. In: Appezzato-da-Glória, B, \& Carmello-Guerreiro, S. M. (eds.). Anatomia vegetal. Viçosa: Editora UFV.

Amorozo, M. C. M., \& Gély, A. (1988). Uso de plantas medicinais por caboclos do Baixo Amazonas. Barcarena, PA, Brasil. Boletim do Museu Emílio Goeldi, Série Botânica, 4(supl.), 47-129.

Andrade, N. D., Almeida, B. M., Souza, R. M. S., \& Araújo, M. S. (2021). Uso das plantas medicinais para fins terapêuticos por estudantes do Ensino Médio. Research, Society and Development, 10(4), e59510414484. doi.org/10.33448/rsd-v10i4.14484

APweb. (2021). Angiosperm phylogeny website. http://www.mobot.org/MOBOT/research/APweb/.

Bandeira, J. M. (2007). Análise da diversidade genética entre genótipos do gênero Plectranthus. Dissertação de Mestrado, Universidade Federal de Pelotas, Pelotas, PR, Brasil.

Barroso, G. M., Morim, M. P., Peixoto, A. L., \& Ichasso, C. L. F. (1999). Frutos e sementes: morfologia aplicada a sistemática de dicotiledôneas. Viçosa: Editora UFV.

Brasil. (2016). Política e programa nacional de plantas medicinais e fitoterápicos. Série B. Brasília: Ministério da Saúde.

Castro, M. A., Bonilha, O. H., Pantoja, L. D. M., Mendes, R. M. S., Edson-Chaves, B., \& Lucena, E. M. P. (2021). Conhecimento etnobotânico dos alunos de Ensino Médio sobre plantas medicinais em Maranguape - Ceará. Research, Society and Development, 10(3), e8910313008. doi.org/10.33448/rsd-v10i313008

Codd, L. E. (1975). Plectranthus (Labiatae) and allied genera in Southern Africa. Bothalia, 11(4), 371-442.

Cruz, G. S., Schuertz, H. F., \& Dias, G. B. (2017). Uso popular do boldo Plectranthus barbatus Andrews (Lamiaceae) como fitoterápico em tratamento de doenças. Health and Diversity, 1(1), 90-95.

Dluzniewski, F. S., \& Müller, N. T. G. (2018). Estudo etnobotânico de plantas medicinais utilizadas no município de Sete de Setembro, Rio Grande do Sul, Brasil. Perspectiva, 42(157), 49-61.

Duarte, M. R., \& Lopes, J. F. (2007). Stem and leaf anatomy of Plectranthus neochilus Schltr., Lamiaceae. Revista Brasileira de Farmacognosia, 17(4), 549556.

Félix-Silva, J., Tomaz, I. M., Silva, M. G., Santos, K. S. C. R., Silva-Júnior, A. A., Carvalho, M. C. R. D., Soares, L. A. L., \& Fernandes-Pedrosa, M. F. (2012). Identificação botânica e química de espécies vegetais de uso popular no Rio Grande do Norte, Brasil. Revista Brasileira de Plantas Medicinais, 14(3), $548-555$.

Fernandes, J. M. (2002). Plantas medicinais de Alta Floresta: com contribuição à etnobotânica. Alta Floresta: Gráfica cidade.

Fernandes, J. M. (2007). Taxonomia e etnobotânica de Leguminosae Adans. em fragmentos florestais e sistemas agroflorestais na Zona da Mata Mineira. Dissertação de Mestrado, Universidade Federal de Viçosa, Viçosa, MG, Brasil. 
Fernandes, J. M. (2011). Ingeae Benth. (Leguminosae, Mimosoideae) no estado de Minas Gerais, Brasil: taxonomia, morfoanatomia de nectários extraflorais e padrões de distribuição geográfica. Tese de Doutorado, Universidade Federal de Viçosa, Viçosa, MG, Brasil.

Fernandes, J. M., Garcia, F.C. P., Amorozo, M. C. M., Siqueira, L. C., Marotta, C. P. B., \& Cardoso, I. M. (2014). Etnobotânica de Leguminosae entre agricultores agroecológicos na Floresta Atlântica, Araponga, Minas Gerais, Brasil. Rodriguésia, 65(2), 539-554. doi.org/10.1590/S2175-78602014000200015

Ferreira, G. C., Hopkins, M. J. G., \& Secco, R. S. (2004). Contribuição ao conhecimento morfológico das espécies de Leguminosae comercializadas no estado do Pará, como "Angelim". Acta Amazonica, 34(2), 219-232.

Fidalgo, O., \& Bononi, V. L. R. (1989). Técnicas de coleta, preservação e herborização do material botânico. São Paulo: Instituto de Botânica.

Flora do Brasil. (2020). Flora do Brasil 2020 - Algas, Fungos e Plantas. Jardim Botânico do Rio de Janeiro. http://floradobrasil.jbrj.gov.br/.

Galbiatti, M. I. (2019). Estudo da anatomia, morfologia e perfil químico do óleo essencial de Plectranthus neochilus Schltr. (Lamiaceae). Dissertação de Mestrado, Universidade Estadual de Campinas, Campinas, SP, Brasil.

Gois, M. A. F., Lucas, F. C. A., Costa, J. C. M., Moura, P. H. B., \& Lobato, G. J M. (2016). Etnobotânica de espécies vegetais medicinais no tratamento de transtornos do sistema gastrointestinal. Revista Brasileira de Plantas Medicinais, 18(2), 547-557.

Herbário Virtual Reflora. (2020). Consulta pública do herbário virtual. http://reflora.jbrj.gov.br/reflora/herbarioVirtual/.

Jabot. (2020). Consulta - Jardim Botânico do Rio de Janeiro. http://rb.jbrj.gov.br/v2/consulta.php.

Leal-Costa, M. V., Teodoro, F. S., Barbieri, C., Santos, L. F. U., \& Sousa, A. L. (2018). Avaliação da qualidade das plantas medicinais comercializadas no Mercado Municipal de Campos dos Goytacazes-RJ. Revista Fitos, 12(2), 127-134.

Lima, I. E. O., Nascimento, L. A. M., \& Silva, M. S. (2016). Comercialização de Plantas Medicinais no Município de Arapiraca-AL. Revista Brasileira de Plantas Medicinais, 18(2), 462-472.

Lorenzi, H., \& Matos, F. J. A. (2002). Plantas medicinais no Brasil: nativas e exóticas. Nova Odessa: Instituto Plantarum de Estudos da Flora.

Lorenzi, H., \& Matos, F. J. A. (2008). Plantas medicinais no Brasil: nativas e exóticas (2ª ed.). Nova Odessa: Instituto Plantarum de Estudos da Flora.

Mariano, X. M. (2015). Avaliação da composição química do óleo essencial de amostras comerciais de boldo-do-chile (Peumus boldus Molina). Dissertação de Mestrado, Universidade Federal do Estado do Rio de Janeiro, Rio de Janeiro, RJ, Brasil.

Martinelli, G., \& Moraes, M. A. (2013). Livro vermelho da flora do Brasil. Rio de Janeiro: Andrea Jakobsson Editora e Instituto de Pesquisas Jardim Botânico do Rio de Janeiro.

Martins, M. B. G., \& Pastori, A. P. (2004). Anatomia foliar com ênfase nos tricomas secretores e análise cromatográfica ao óleo essencial de Melissa officinalis L. (Lamiaceae). Revista Brasileira de Plantas Medicinais, 6(2), 77-82.

Mera, J. C. E., Rosas, L. V., Lima, R. A., \& Pantoja, T. M. A. (2018). Conhecimento, percepção e ensino sobre plantas medicinais em duas escolas públicas no município de Benjamin Constant - AM. Experiências em Ensino de Ciências, 13(2), 62-79.

Morais, G. F., Oliveira, R. E., Sais, A. C., \& Ramos-Filho, L. O. (2018). Agrobiodiversidade manejada em quintais agroflorestais urbanos: cultivo de plantas medicinais. Cadernos de Agroecologia, 13(2), 1-10.

Moutinho, V. H. P. Caracterização das madeiras conhecidas na Amazônia brasileira como matá-matá (Lecythidaceae fam. A. Rich.). Dissertação de Mestrado, Universidade Federal de Lavas, Lavras, MG, Brasil.

Nakajima, J. (2020). Gymnanthemum in Flora do Brasil 2020. Jardim Botânico do Rio de http://floradobrasil.jbrj.gov.br/reflora/floradobrasil/FB22218.

Pasa, M. C., Hanazaki, N., Silva, O. M. D., Agostinho, A. B., Zank, S., \& Esteves, M. I. P. N. (2019). Medicinal plants in cultures of Afro-descendant communities in Brazil, Europe and Africa. Acta Botanica Brasilica, 33(2), 340-349. https://doi.org/10.1590/0102-33062019abb0163

Pereira, M. G. S., \& Coelho-Ferreira, M. (2017). Uso e diversidade de plantas medicinais em uma comunidade quilombola na Amazônia Oriental, Abaetetuba, Pará. Biota Amazônia, 7(3), 57-68.

Radford, A. E., Dickison, W. C., Massey, J. R., \& Bell, C. R. (1974). Vascular plant systematics. New York: Harper \& Row.

Rodrigues, V. G. S. (2004). Cultivo, uso e manipulação de plantas medicinais (série 91): Embrapa.

Schlechter, F. R. R. (1896). Decades plantarum novarum austro-africanarum. Journal of Botany, British and Foreign, 34, $391-395$.

Silva, A. C. M., Leite, R. S., Yoshida, E. H., Carneiro, H. F. P., \& Santos, N. S. (2019). O uso de três plantas medicinais populares no Brasil: uma revisão da literatura. Revista Saúde em Foco, 1(1), 435-444.

Souza, C. M. P., Brandão, D. O., Silva, M. S. P., Palmeira, A. C., Simões, M. O. S., \& Medeiros, A. C. D. (2013). Utilização de plantas medicinais com atividade antimicrobiana por usuários do serviço público de saúde em Campina Grande - Paraíba. Revista Brasileira de Plantas Medicinais, 15 (2), 188-193.

Souza, M. R. M., Pereira, R. G. F., \& Fonseca, M. C. M. (2012). Comercialização de plantas medicinais no contexto da cadeia produtiva em Minas Gerais. Revista Brasileira de Plantas Medicinais, 14(n.esp.), 242-245.

SpeciesLink. (2020). SpeciesLink network. http://www.splink.org.br/. 
Research, Society and Development, v. 10, n. 6, e42910615824, 2021

(CC BY 4.0) | ISSN 2525-3409 | DOI: http://dx.doi.org/10.33448/rsd-v10i6.15824

Swelankomo, N., Manning, J. C., \& Magee, A. R. (2016). The genus Gymnanthemum Cass. (Asteraceae: Vernonieae) in Southern Africa. South African Journal of Botany, 102, 81-101. doi.org/10.1016/j.sajb.2015.07.015

Tropicos. (2020). Connecting the world to botanical data since 1982. https://www.tropicos.org/home.

Vasconcelos, A. L. F. C., Morais, S. M., Santos, L. F. L., Rocha, M. F. G., Bevilaqua, \& C. M. L. (2005). Validação de plantas medicinais com atividade antihelmíntica. Revista Brasileira de Plantas Medicinais, 7(3), 97-106.

World Flora Online. (2021). An online flora of all known plants: supporting the global strategy for plant conservation. http://www.worldfloraonline.org/. 